PROCEEDINGS OF THE

AMERICAN MATHEMATICAL SOCIETY

Volume 126, Number 10, October 1998, Pages 3079-3082

S 0002-9939(98)04629-2

\title{
A VOLUME COMPARISON THEOREM FOR FINSLER MANIFOLDS
}

\author{
CARLOS E. DURÁN
}

(Communicated by Christopher B. Croke)

\begin{abstract}
Let $\left(M^{n}, F\right)$ be a symmetric Finsler manifold, endowed with the Busemann volume form, and let $D$ be its unit disk bundle endowed with the canonical symplectic volume form. It is shown that $\operatorname{Vol}(D) \leq C(n) \operatorname{Vol}\left(M^{n}\right)$, where $C(n)$ is the volume of the unit disk in $\mathbb{R}^{n}$. Moreover, equality holds if and only if $\left(M^{n}, F\right)$ is Riemannian.
\end{abstract}

\section{INTRODUCTION}

A Finsler structure on a manifold $M^{n}$ is a function $F: T M \rightarrow \mathbb{R}$ which is homogenous of degree 1, strictly convex and smooth off the zero section. The function $F$ is to be thought of as a "norm" in each tangent space $T_{p} M$, which is not necessarily Euclidean. The Finsler manifold $(M, F)$ is said to be symmetric if $F(v)=F(-v)$.

There has been a revived interest in the study of Finsler manifolds recently, sparked by S.S. Chern and others, (e.g., [2], [3], [9]). There are also some older, beautiful geometric studies of Finsler manifolds; for example, [1], [5], [6], [7]. A basic problem is to find computable invariants that distinguish Finsler manifolds from Riemannian manifolds; the basic invariant is the Cartan 3-tensor $A$, which is, in essence, simply three derivatives of the energy function along the fibers. Riemannian manifolds are characterized by $A \equiv 0$. (see [2]).

In this note we will find a global integral invariant of Finsler metrics that attains its maximum exactly at the set of Riemannian metrics: the ratio of the symplectic volume of the unit disk bundle to the volume of the manifold. The philosophy behind the construction is that of Busemann: Finsler geometry should be thought of as the geometry of families of convex sets in $\mathbb{R}^{n}$, parametrized by a manifold. These geometries can be put together via the associated calculus of variations, i.e. the Hamiltonian geometry of $(T M, \omega, F)$, where $\omega$ is the pullback of the canonical symplectic form of $T^{*} M$ via the Legendre transformation.

\section{The Busemann volume Form}

Let $(M, F)$ be a symmetric Finsler manifold. The Busemann volume form of $(M, F)$ is defined as follows: let $e^{1}, \ldots, e^{n}$ be a basis for $T_{x} M$, with dual basis

Received by the editors March 6, 1997.

1991 Mathematics Subject Classification. Primary 53C60, 53C15.

Key words and phrases. Riemannian geometry, Finsler geometry.

Supported by CNPq, Brasil.

(C)1998 American Mathematical Society 
$\eta_{1}, \ldots, \eta_{n}$. Given $x \in M$, let $D(x) \subset \mathbb{R}^{n}$ be given by

$$
D(x)=\left\{y \in \mathbb{R}^{n}: F\left(y_{i} e^{i}\right) \leq 1\right\}
$$

(summation convention will be used throughout). Denote by $V(x)$ the volume of $D(x)$, with respect to the standard Euclidean structure of $\mathbb{R}^{n}$. Then the Busemann volume form $B_{F}$ is given by

$$
B_{F}=\frac{C(n)}{V(x)} \eta_{1} \wedge \ldots \wedge \eta_{n}
$$

where $C(n)$ is the volume of the unit disk in $\mathbb{R}^{n}$. It is easy to see that $B$ does not depend on the choice of the (positively oriented) basis. The induced measure on $M$ coincides with the Hausdorff measure given by the metric; thus $B_{F}$ is in the metric sense the "right" volume form for a Finsler manifold (see [4], [9]). As usual, we define the volume of $M$ by

$$
\operatorname{vol}(M)=\int_{M} B_{F}
$$

On the other hand, the punctured tangent bundle $T_{0} M$ has a symplectic form $\omega$, given by the pullback of the canonical symplectic form of $T^{*} M$ by the Lagrange transformation $v \mapsto L_{F}(v)$. The top form $\frac{(-1)^{n}}{(2 n) !} \omega^{n}$ is the canonical volume form on $T_{0} M$. In local coordinates $v=\left(x^{i}, y_{i} \frac{\partial}{\partial x^{i}}\right)$ of $T M$, we can write

$$
L(x, y)=\frac{1}{2} \frac{\partial F^{2}}{\partial y_{i}} d x^{i}, \quad \frac{(-1)^{n}}{(2 n) !} \omega^{n}=\operatorname{det}\left(g_{i j}\right) d x^{1} \wedge \cdots \wedge d x^{n} \wedge d y_{1} \cdots \wedge d y_{n},
$$

where $g_{i j}=\frac{1}{2} \frac{\partial^{2} F^{2}}{\partial y_{i} \partial y_{j}}$ is called the fundamental tensor of the Finsler metric $F$.

Let $C(n)$ and $c(n)$ be the volume of the unit disk and the unit sphere in $\mathbb{R}^{n}$, respectively. Then we have

Theorem 1. Let $D$ be the unit disk in $T_{0} M$, and let $\operatorname{Vol}(D)$ be its volume with respect to the symplectic form. Then

$$
\operatorname{Vol}(D) \leq C(n) \operatorname{vol}(M),
$$

with equality if and only if $(M, F)$ is Riemannian.

Recall that $\omega=-d \alpha$ where $\alpha$ is the pullback by the Lagrange transformation of the canonical 1-form of $T^{*} M$. Then the unit tangent bundle $\{F=1\}$ has an exact contact structure given by $\alpha$, and corresponding volume form $\frac{1}{(n-1) !} \alpha \wedge(d \alpha)^{n-1}$. We have then the corresponding theorem for the unit tangent bundle:

Corollary. Let $S$ be the unit tangent bundle $\{F=1\}$, and let $\operatorname{Vol}(S)$ be its volume with respect to the contact structure. Then

$$
\operatorname{Vol}(S) \leq c(n) \operatorname{vol}(M)
$$

with equality if and only if $(M, F)$ is Riemannian.

\section{Proof of Theorem 1}

Cover $M$ with a full measure coordinate patch giving us coordinates $(x, y)$ on $T M$. Then

$$
\omega^{n}=\operatorname{det}\left(g_{i j}\right) d x^{1} \wedge \ldots \wedge d x^{n} \wedge d y_{1} \wedge \ldots \wedge d y_{n},
$$

which we write in the more compact notation $\operatorname{det}\left(g_{i j}\right) d x \wedge d y$. 
Integrating over the fiber, we have

$$
\int_{D} \omega=\int_{x \in M} \int_{D_{x}} \omega=\int_{x \in M} d x \int_{D(x)} \operatorname{det}\left(g_{i j}\right)(x, y) d y .
$$

Let $D^{*}(x)$ be the dual of the convex set $D(x)$. Recall that given a convex set $K$ in a vector space $V$, the dual set $K^{*} \subset V^{*}$ is defined by

$$
K^{*}=\left\{\xi \in V^{*}: \xi(v) \leq 1 \forall v \in K\right\}
$$

(see the beautiful book [10] for more details on duality and convex geometry in general).

Note that the map

$$
y_{i} \partial_{x^{i}} \mapsto \frac{1}{2} \frac{\partial F^{2}}{\partial y_{i}}(y) d x^{i}
$$

is a diffeomorphism between $D(x)$ and $D^{*}(x)$, with Jacobian $\operatorname{det}\left(g_{i j}\right)$. Therefore,

$$
\int_{D(x)} \operatorname{det}\left(g_{i j}\right)(x, y) d y=\int_{D^{*}(x)} d y=\operatorname{Vol}\left(D^{*}(x)\right) .
$$

Integrating over $M$, we have

$$
\operatorname{Vol}(D)=\int_{M} \operatorname{Vol}\left(D^{*}(x)\right) d x=\frac{1}{C(n)} \int_{M} C(n) \operatorname{Vol}\left(D^{*}(x)\right) d x .
$$

Let $\mu(x)=\operatorname{Vol}(D(x)) \operatorname{Vol}\left(D^{*}(x)\right)$. The volume product $\mu$ is an affine invariant of the convex body $D(x)$, and we have Santaló's inequality (see [8] and the references therein)

$$
\mu(x) \leq \operatorname{Vol}(E) \operatorname{Vol}\left(E^{*}\right)=C(n)^{2},
$$

where $E$ is any ellipsoid. Moreover, equality occurs if and only if $D(x)$ is an ellipsoid.

Then we have

$$
\begin{aligned}
\operatorname{Vol}(D) & =\int_{M} \operatorname{Vol}\left(D^{*}(x)\right) d x \\
& =\frac{1}{C(n)} \int_{M} \frac{C(n) \mu(x)}{\operatorname{Vol}(D(x))} d x \\
& =\frac{1}{C(n)} \int_{M} \mu(x) B_{F} \\
& \leq C(n) \operatorname{vol}(M)
\end{aligned}
$$

which proves the inequality in Theorem 1. Equality occurs if and only if $\mu(x) \equiv$ $C(n)^{2}$ which only happens if for each $x, F(x, y) \leq 1$ is the interior of an ellipsoid symmetric with respect to the origin, which means that the metric is Riemannian.

The corollary follows from Theorem 1 and Stokes's Theorem.

Note that the proof of Theorem 1 and the non-symmetric version of Santalós inequality also show the following for non-symmetric Finsler manifolds:

Let $\left(M^{n}, F\right)$ be a non-symmetric Finsler manifold such that the centroid of the unit disk coincides with zero. Then $\operatorname{Vol}(D) \leq C(n) \operatorname{Vol}(M)$, with equality if and only if the unit disk is an ellipsoid. Therefore such a Finsler metric is actually symmetric and Riemannian. 


\section{REFERENCES}

1. H. Akbar-Zadeh, Sur les espaces de Finsler a courbures sectionnelles constantes, Acad. Roy. Belg. Bull. Cl. Sci 74 (1988), 281-322. MR 91f:53069

2. D. Bao, S.-S. Chern and Z. Shen, An Introduction to Riemann-Finsler Geometry, SpringerVerlag, Berlin.

3. R. Bryant, Finsler structures on the 2-sphere satisfying $K=1$, Contemporary Mathematics, vol. 196, 1996, pp. 27-41. MR 97e:53128

4. H. Busemann, Intrinsic Area, Ann. Math. 48 (1947), 234-267. MR 8:573a

5. H. Busemann, The geometry of Finsler spaces, Bull. Amer. Math. Society 56 (1950), 5-16. MR 11:400e

6. H. Busemann, Geometry of Geodesics, Academic Press, New York, 1955. MR 17:779a

7. E. Cartan, Sur un problème d'equivalence et la théorie des espaces métriques generalisés, Ouvres Complétes, Vol. II, Part. III, Gauthier-Villars, Paris, pp. 1131-1153.

8. M. Meyer and A. Pajor, On Santaló's inequality, Geometric aspects of functional analysis, Springer Lecture Notes in Mathematics 1376, pp. 261-263. MR 90h:52012

9. Z. Shen, On Finsler geometry of submanifolds, Preprint (1996).

10. A.C. Thompson, Minkowski Geometry, Cambridge University Press, Cambridge, 1996. MR 97f:52001

impa, Estrada Dona Castorina 110, Jardim Botânico, Rio de Janerio RJ 22460-320, BRASIL

Current address: IVIC-Matematicas, Apartado 21827, Caracas 1020-A, Venezuela

E-mail address: cduran@impa.br, cduran@cauchy.ivic.ve 